\title{
LA ENCUESTA DE FECUNDIDAD DE 1977 \\ DEL INSTITUTO NACIONAL DE ESTADISTICA
}

\author{
Jesús M. de Miguel \\ (Universitat Autònoma de Barcelona)
}

En el año 1977 el Instituto Internacional de Estadística, bajo el patrocinio de la Organización de las Naciones Unidas, promovió un estudio comparativo sobre pautas de fecundidad y control de natalidad en diversos países. El Instituto Nacional de Estadistica (INE) español se encargó de la parte referente a nuestro país, diseñando una encuesta a $5.814 \mathrm{mu}-$ jeres, casadas-alguna-vez (es decir, casadas, viudas, divorciadas y separadas), en edad teóricamente fértil, de 15 a 49 años de edad. Se trataba pues de las cohortes de mujeres nacidas entre 1927 y 1962.

Las entrevistas se tealizaron entre los meses de noviembre y diciembre de 1977, y el informe final Encuesta de fecundidad: Metodología y resultados fue publicado en mayo de 1978 (con 227 páginas, más anexos). Se editó además un folleto - de supuesta difusión popular- con algunos de los datos más importantes: La fecundidad en España: Diciembre 1977 (Madrid: Instituto Nacional de Estadística, 1978), 45 páginas. Salvo ese breve comentario de la citada encuesta sólo se publicaron las tablas estadísticas (y no todas), sin un análisis posterior. Hasta la fecha, la encuesta de fecundidad del INE es el estudio más completo e importante que existe en España sobre pautas de control de natalidad, y fecundidad. Desgracia. damente todavía no ha sido analizado en profundidad por la profesión sociológica.

La citada encuesta internacional, a pesar de los «retoques» de que fue objeto en España, se vio atacada desde los sectores más conservadores. Según el propio informe: «El compromiso a que había llegado la Dirección 
del INE fue visto con recelo por determinados grupos sociales que iniciaron una campaña contra esta investigación esgrimiendo argumentos legales y mediante una campaña de prensa» (p. 8). De todas maneras no se pudieron incluir preguntas directas sobre métodos concretos de control de natalidad si no eran mencionados por la entrevistada, pues en aquellos momentos podía todavía considerarse como un delito de propaganda inmoral. A pesar de todo se continuó afirmando que la encuesta iba a «constituir un asalto a la vida íntima de los matrimonios con objeto de divulgar unos métodos que incluso podrían acarrear descomposición familiars (p. 10).

La población total a investigar (el universo) supone 5,2 millones de mujeres "no solteras», y se obtuvieron 1,1 entrevistas por cada $1.000 \mathrm{mu}-$ jeres (no solteras) de 15 a 49 años de edad, de todas las áreas geográficas de España. Aún, cuando me refiero a «mujeres-no-solteras» tan sólo un $2,2 \%$ de la muestra son viudas, y un $0,9 \%$ son divorciadas o separadas. Por ello, prefiero utilizar en sentido amplio «mujeres-casadas», pues el $97 \%$ de la muestra está casada todavía, y generalmente sólo una vez. Tan sólo un $0,4 \%$ de la muestra se refiere a mujeres que han estado dos veces casadas, y no hay casos de tres o más matrimonios. Tan sólo un $1,0 \%$ de los matrimonios actuales son entre soltera y viudo; el $0,5 \%$ entre viuda y viudo; y el $0,3 \%$ entre viuda y soltero (a pesar de que hay más viudas que viudos).

En las tablas que siguen elaboto algunos de los datos más importantes, a partir de las estadísticas originales de la Encuesta de Fecundidad de 1977. Estos materiales de investigación son parte de un informe completo sobre población y control de natalidad en España, en prensa.*

* Agradezco la ayuda técnica de Lourdes Rubio Vergés y los comentarios y críticas de José L. Crespán. 
La encuesta cle fecundidad de 1977 del INE

TABLA 1

Conocimiento y utilización de métodos de contracepción en España (1977)

\begin{tabular}{|c|c|c|c|}
\hline \multirow[b]{2}{*}{ Métodos de contracepción } & \multicolumn{3}{|c|}{ Porcentajes de mujeres casadas } \\
\hline & $\begin{array}{l}\text { Conocen } \\
\text { el método }\end{array}$ & $\begin{array}{l}\text { Lo ban utilizado } \\
\text { alguna vez }\end{array}$ & $\begin{array}{l}\text { Lo utilizan en } \\
\text { la actualidad }\end{array}$ \\
\hline DIU ... & $30 \%$ & $0,6 \%$ & $0,5 \%$ \\
\hline Píldora & $88 \%$ & $17,0 \%$ & $12,0 \%$ \\
\hline Diafragma $\ldots \ldots \ldots \ldots \ldots \ldots$ & $19 \%$ & $0,1 \%$ & $0,1 \%$ \\
\hline Condón $\ldots \ldots \ldots \ldots \ldots \ldots$ & $68 \%$ & $7,0 \%$ & $5,0 \%$ \\
\hline Ritmo $\ldots \ldots \ldots \ldots \ldots \ldots \ldots$ & $53 \%$ & $13,0 \%$ & $6,0 \%$ \\
\hline Coito interrumpido .......... & $70 \%$ & $31,0 \%$ & $23,0 \%$ \\
\hline Lactancia prolongada ....... & $34 \%$ & $0,4 \%$ & $0,0 \%$ \\
\hline Lavado vaginal . .............. & $36 \%$ & $1,0 \%$ & $0,6 \%$ \\
\hline Ninguno $. . . \ldots \ldots \ldots \ldots \ldots . . . . .$. & $7 \%$ & $40,0 \%$ & $47,0 \%$ \\
\hline Total $\ldots \ldots \ldots \ldots \ldots \ldots$ & $(5.814)$ & $(5.814)$ & $(5.315)$ \\
\hline
\end{tabular}

Notas: a) No incluye fas embarazadas. Actuajmente (un $6 \%$ de la muestra); pero sí incluye las que acaban de dar a luz y están todavia en el post-partum y las que están esperando quedar embarazadas (se podría calcular en un $8 \%$ a ur $10 \%$ adicional); y el porcentaje de ratujeres estériles que no pueden tener descendencia $(5 \%$ al $10 \%)$. Es decir, entre e) 19 \%6 y el $26 \%$ del total de mujeres de 15 a 49 años no necesitan controlar la natalidad. Fuente: INE, Encuesta de fecundidad (Madrid: INE, 1978), pp. 156, 163 y 180. 
«Papers»: Revista de Sociologia

\section{TABLA 2}

Utilización de métodos de contracepción por las mujeres casadas menores de 25 af́os

\begin{tabular}{|c|c|c|c|}
\hline \multirow[b]{2}{*}{ Métodos de contracepción } & \multicolumn{3}{|c|}{$\begin{array}{l}\text { Porcentaje de mujeres casadas } \\
\text { (menores de } 25 \text { años de edad) }\end{array}$} \\
\hline & $\begin{array}{l}\text { Conocen } \\
\text { el método }\end{array}$ & $\begin{array}{l}\text { Lo ban utilizado } \\
\text { alguna vez }\end{array}$ & $\begin{array}{l}\text { Lo utilizan en } \\
\text { la actualidad }\end{array}$ \\
\hline DIU $\quad \ldots \ldots \ldots \ldots \ldots \ldots \ldots \ldots$ & $41 \%$ & $1,4 \%$ & $\ldots{ }^{b}$ \\
\hline 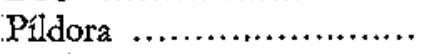 & $97 \%$ & $33,0 \%$ & $23,0 \%$ \\
\hline Diafragma $\ldots \ldots \ldots \ldots \ldots \ldots$ & $28 \%$ & 一 & $\cdots$ \\
\hline 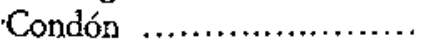 & $79 \%$ & $8,0 \%$ & $\cdots$ \\
\hline Ritmo ...................... & $57 \%$ & $5,0 \%$ & ... \\
\hline Coito interrumpido ......... & $78 \%$ & $24,0 \%$ & $\ldots$ \\
\hline Lactancia prolongada ....... & $38 \%$ & - & $\cdots$ \\
\hline Lavado vaginal ............. & $46 \%$ & $1,4 \%$ & $\cdots$ \\
\hline Ninguno..$\ldots \ldots \ldots \ldots \ldots \ldots$ & $2 \%$ & $32,0 \%$ & $41,0 \%$ \\
\hline Total $\ldots \ldots \ldots \ldots \ldots \ldots$ & $(695)$ & $(695)$ & $(597)$ \\
\hline
\end{tabular}

Notas: a) No incluye las embarazadas.

b) El INE no ofrece estos datos en sus tablas.

Fuente: INE, Encuesta de fecundidad (Madrid: INB, 1978), pp. 156, 163, 187 y 188. 


\section{TABLA 3}

Proporción de mujeres qué controlan la natalidad, según el número de hijos tenidos

\begin{tabular}{|c|c|c|c|c|}
\hline \multirow[b]{2}{*}{$\begin{array}{l}\text { Número de bijas } \\
\text { e bijos tenidos }\end{array}$} & \multicolumn{3}{|c|}{$\%$ de mujeres casadas que } & \multirow[b]{2}{*}{ Total } \\
\hline & $\begin{array}{l}\text { Nunca ban } \\
\text { controlado } \\
\text { la natalidad }\end{array}$ & $\begin{array}{l}\text { Han controlado } \\
\text { pero no en } \\
\text { la actualidad }\end{array}$ & $\begin{array}{l}\text { Controlan } \\
\text { actualmente } \\
\text { la natalidad }\end{array}$ & \\
\hline Ninguno .......... & $78 \%$ & $6 \%$ & $16 \%$ & $100 \%(334)$ \\
\hline Uno $\ldots \ldots \ldots \ldots . . .$. & $41 \%$ & $12 \%$ & $48 \%$ & $\{1.203\}$ \\
\hline Dos $\ldots \ldots \ldots \ldots \ldots$ & $36 \%$ & $11 \%$ & $53 \%$ & $(2.054)$ \\
\hline Tres $\ldots . . . . . . . . . .$. & $35 \%$ & $13 \%$ & $52 \%$ & $(1.170)$ \\
\hline Cuatro ............... & $36 \%$ & $11 \%$ & $53 \%$ & $(580)$ \\
\hline Cinco o más ... & $42 \%$ & $14 \%$ & $44 \%$ & (473) \\
\hline Total ........ & $39 \%$ & $11 \%$ & $49 \%$ & $(5.814)$ \\
\hline
\end{tabular}

Fuente: INE, Encuesta de fecundidad (Madrid: INE, 1978), p. 195.

\section{TABLA 4}

Métodos de contracepción utilizados según el número de hijos

\begin{tabular}{|c|c|c|c|c|}
\hline \multirow{2}{*}{$\begin{array}{c}\text { Método de contracepción } \\
\text { utilizado actualmente }\end{array}$} & \multicolumn{4}{|c|}{ Número de bijas e bijos tenidos } \\
\hline & Ninguno & Uno & Dos & Tres y más \\
\hline Coito interrumpido ... & $0,6 \%$ & $16,0 \%$ & $24,0 \%$ & $24,0 \%$ \\
\hline Píldora .................... & $6,0 \%$ & $16,0 \%$ & $12,0 \%$ & $9,6 \%$ \\
\hline Ritmo $\ldots \ldots \ldots \ldots \ldots \ldots$ & $1,5 \%$ & $4,6 \%$ & $6,4 \%$ & $6,3 \%$ \\
\hline Condón,$\ldots \ldots \ldots \ldots \ldots \ldots$ & $0,0 \%$ & $4,4 \%$ & $4,5 \%$ & $5,2 \%$ \\
\hline Otros métodos ............. & $1,2 \%$ & $3,6 \%$ & $3,9 \%$ & $4,2 \%$ \\
\hline Total .... & $\begin{array}{r}9,3 \% \\
(334)\end{array}$ & $\begin{array}{l}40,0 \% \\
(1.203)\end{array}$ & $\begin{array}{l}50,0 \% \\
(2.054)\end{array}$ & $\begin{array}{l}49,0 \% \\
(2.223)\end{array}$ \\
\hline
\end{tabular}

Nota: Incluye tan solo las mujeres casadas actualmente, y expusstas al riesgo del embarazo, que utilizan metodos contraceptivos. Difiere, pues, de los totales de la Tabla 3 que se refiere a todas las mujeres no solteras, e incluye los casos de esterilizacion.

Fuenteः INE, Encuesta de fecundidad (Madrid: INE, 1978), p. 174. 
«Papers»: Revista de Sociologia

\section{TABLA 5}

Proporción de mujeres casadas que controlan la natalidad, según la edad de la mujer

\begin{tabular}{|c|c|c|c|c|}
\hline \multirow[b]{2}{*}{$\begin{array}{l}\text { Grupo de edad } \\
\text { de la mujer }\end{array}$} & \multicolumn{4}{|c|}{$\%$ de mujeres casadas } \\
\hline & $\begin{array}{l}\text { Nunca ban } \\
\text { controlado } \\
\text { la natalidad }\end{array}$ & $\begin{array}{c}\text { Han } \\
\text { controlado, } \\
\text { pero no en la } \\
\text { actualidad }\end{array}$ & $\begin{array}{l}\text { Controlan } \\
\text { actualmente } \\
\text { la natalidad }\end{array}$ & Total \\
\hline Menores de 25 años. & $33 \%$ & $13 \%$ & $53 \%$ & $100 \%(695)$ \\
\hline 25 a 29 años ...... & $34 \%$ & $9 \%$ & $57 \%$ & $(1.030)$ \\
\hline 30 a 34 años $\ldots . .$. & $31 \%$ & $11 \%$ & $58 \%$ & $(1.033)$ \\
\hline 35 a 39 años ..... & $38 \%$ & $9 \%$ & $53 \%$ & (924) \\
\hline 40 a 44 años ..... & $43 \%$ & $11 \%$ & $46 \%$ & $(1.071)$ \\
\hline 45 años y más ..... & $54 \%$ & $15 \%$ & $31 \%$ & $(1.061)$ \\
\hline Total ............. & $39 \%$ & $11 \%$ & $49 \%$ & $(5.814)$ \\
\hline
\end{tabular}

Nota: Del total se han descontado los casos en que no consta información acerca del control, aun cuando suponemos que controlan la natalidad muy por debajo de la media.

Fuente: INE, Encuesta de fecundidad (Madrid: INE, 1978), p. 192.

TABLA 6

Métodos de contracepción utilizados según la edad de la mujer

\begin{tabular}{|c|c|c|c|c|}
\hline \multirow[b]{2}{*}{$\begin{array}{l}\text { Métodos de contracepción } \\
\text { que utilizan en la actualidad }\end{array}$} & \multicolumn{4}{|c|}{$\%$ de mujeres (casadas) que controlan } \\
\hline & $\begin{array}{l}\text { Menores de } \\
30 \text { años }\end{array}$ & $\begin{array}{l}\text { De } 30 \text { a } \\
39 \text { años }\end{array}$ & $\begin{array}{l}\text { De } 40 y \\
\text { más años }\end{array}$ & Total \\
\hline Coito interrumpido & $18,0 \%$ & $25,0 \%$ & $13,0 \%$ & $21,0 \%$ \\
\hline Píldora............. & $20,0 \%$ & $11,0 \%$ & $2,2 \%$ & $11,0 \%$ \\
\hline Ritmo $\ldots \ldots \ldots \ldots \ldots \ldots \ldots \ldots$ & $4,1 \%$ & $7,8 \%$ & $3,4 \%$ & $5,7 \%$ \\
\hline Condón $. . . \ldots \ldots \ldots \ldots \ldots . . . . . . .$. & $5,3 \%$ & $5,2 \%$ & $2,2 \%$ & $4,5 \%$ \\
\hline Otros métodos ............... & $5,2 \%$ & $4,1 \%$ & $1,7 \%$ & $3,8 \%$ \\
\hline Total ... & $\begin{array}{l}52,0 \% \\
(1.725)\end{array}$ & $\begin{array}{l}53,0 \% \\
(1.957)\end{array}$ & $\begin{array}{l}23,0 \% \\
(2.132)\end{array}$ & $\begin{array}{l}46,0 \% \\
(5.814)\end{array}$ \\
\hline
\end{tabular}

Nota: AI igual que otras tablas sobre métodos de contracepción utilizados» se refiere a mu. jeres actualmente casadas y expuestas al riesgo del embarazo. No inciuye, pues, parejas esterilizadas.

Fuente: INE, Encuesta de fecundidad (Madrid: INE, 1978), pp. 155, 168 y 169. 
La encuesta de fecundidad de 1977 del INE

TABLA 7

Métodos de contracepción utilizados según la religiosidad de la mujer

\begin{tabular}{|c|c|c|c|c|}
\hline \multirow[b]{2}{*}{$\begin{array}{l}\text { Método de contracepción } \\
\text { utilizado actualmente }\end{array}$} & \multicolumn{4}{|c|}{ Religiosidad de la mujer } \\
\hline & $\begin{array}{l}\text { Católica } \\
\text { practicante }\end{array}$ & $\begin{array}{l}\text { Católica no } \\
\text { practicante }\end{array}$ & $\begin{array}{l}\text { Creyente de } \\
\text { otra religión }\end{array}$ & $\begin{array}{l}\text { No } \\
\text { creyente }\end{array}$ \\
\hline Coito interrumpido ....... & $21,0 \%$ & $20,0 \%$ & $22,0 \%$ & $17,0 \%$ \\
\hline Pildora $\ldots \ldots \ldots \ldots \ldots \ldots \ldots$ & $7,8 \%$ & $21,0 \%$ & $13,0 \%$ & $20,0 \%$ \\
\hline Ritmo $\ldots \ldots \ldots \ldots \ldots \ldots \ldots$ & $6,4 \%$ & $4,0 \%$ & $2,2 \%$ & $2,0 \%$ \\
\hline Condón $\ldots . . . \ldots \ldots \ldots . . . . . .$. & $4,2 \%$ & $6,1 \%$ & $5,5 \%$ & $2,3 \%$ \\
\hline Otros métodos ............ & $3,2 \%$ & $6,1 \%$ & $1,1 \%$ & $7,0 \%$ \\
\hline Total ....... & $\begin{array}{l}43,0 \% \\
(4.463)\end{array}$ & $\begin{array}{r}57,0 \% \\
(961)\end{array}$ & $\begin{array}{r}44,0 \% \\
(91)\end{array}$ & $\begin{array}{r}49,0 \% \\
(300)\end{array}$ \\
\hline
\end{tabular}

Fuente: INE, Encuesta de fecundidad (Madrid: INE, 1978). p. 175.

TABLA 8

Utilización de métodos de contracepción por las mujeres católicas (casadas), en España y los Estados Unidos

\begin{tabular}{|c|c|c|c|c|}
\hline \multirow[b]{2}{*}{ Métodos de control } & \multicolumn{2}{|c|}{ España, 1977} & \multicolumn{2}{|c|}{$\mathrm{USA}, 1973$} \\
\hline & Total & $\begin{array}{c}\text { Católicas } \\
\text { practicantes }\end{array}$ & Total & Católicas \\
\hline Píldora & $12,0 \%$ & $7,8 \%$ & $25,0 \%$ & $23,0 \%$ \\
\hline Condón $\ldots . . . . . . . . . . . .$. & $4,9 \%$ & $4,2 \%$ & $9,4 \%$ & $10,0 \%$ \\
\hline Ritmo $\ldots . . . . . . . . . . . . .$. & $6,3 \%$ & $6,4 \%$ & $2,8 \%$ & $5,5 \%$ \\
\hline Coito interrumpido ..... & $23,0 \%$ & $21,0 \%$ & $1,5 \%$ & $1,9 \%$ \\
\hline Otros sistemas ........... & $6,8 \%$ & $3,2 \%$ & $31,0 \%=$ & $26,0 \% \mathrm{~b}$ \\
\hline Ninguno ................... & $47,0 \%$ & $57,0 \%$ & $30,0 \%$ & $34,0 \%$ \\
\hline Total $\ldots \ldots \ldots \ldots \ldots$ & $\begin{array}{l}100 \% \\
(5.315)\end{array}$ & $(4.463)$ & $(26.646)$ & $(7.661)$ \\
\hline$\%$ respecto del total ... & - & $84,0 \%$ & - & $29,0 \%$ \\
\hline
\end{tabular}

Notas: a) Fundamentalmente: estelilización (16\%), DIU (7\%), espuma (3 \%) y diafragma (2\%).

b) Fundamentalmente: esteritizacion (18 \%), DIU (6 \%), espuma (3\%), y diafragma (2\%).

Fuentes: INE, Encuesta de fecundidad (Madrid: INE, 1978), pp. 175 y 180.

Kathleen Ford, Contraceptive Utitization in the United States (Hyattsvilte, Md.: National Center for Health Statistics, 1979), pp. 14, 31 y 32. 
«Papers»: Revista de Sociologia

\section{TABLA 9}

Proporción de mujeres casadas que controlan la natalidad según el nivel de estudios de la mujer

\begin{tabular}{|c|c|c|c|c|}
\hline \multirow[b]{2}{*}{ Estudios de la mujer } & \multicolumn{3}{|c|}{$\%$ de mujeres que ${ }^{\mathrm{a}}$} & \multirow[b]{2}{*}{ Total ${ }^{b}$} \\
\hline & $\begin{array}{c}\text { Nunca ban } \\
\text { controlado } \\
\text { la } \\
\text { natalidad }\end{array}$ & $\begin{array}{l}\text { Han } \\
\text { controlado, } \\
\text { pero no } \\
\text { en la } \\
\text { actualidad }\end{array}$ & $\begin{array}{l}\text { Controlan } \\
\text { actualmente } \\
\text { la natalidad }\end{array}$ & \\
\hline Superiores .............. & $17 \%$ & $19 \%$ & $63 \%$ & $100 \%(68)$ \\
\hline Medios,$\ldots \ldots \ldots \ldots \ldots . . .$. & $18 \%$ & $17 \%$ & $64 \%$ & (198) \\
\hline Bachillerato superior... & $21 \%$ & $20 \%$ & $60 \%$ & $(232)$ \\
\hline Bachillerato elemental & $25 \%$ & $15 \%$ & $60 \%$ & (392) \\
\hline Primaria...$\ldots \ldots \ldots \ldots$ & $40 \%$ & $10 \%$ & $49 \%$ & $(3.353)$ \\
\hline Sin estudios $\ldots \ldots \ldots \ldots$ & $46 \%$ & $10 \%$ & $43 \%$ & $(1.053)$ \\
\hline Analfabetas,$\ldots \ldots \ldots \ldots$ & $48 \%$ & $12 \%$ & $40 \%$ & (515) \\
\hline Total $\ldots \ldots \ldots \ldots \ldots$ & $39 \%$ & $11 \%$ & $49 \%$ & $100 \%(5.810)$ \\
\hline
\end{tabular}

Notas: a) Técnicamente mujeres no solteras.

b) No se incluyen los casos en que no consta la información sobre control de natalidad.

Fuente: INE, Encuesta de fecundided (Madrid: INE, 1978), p. 194. 
La encuesta de fecundidad de 1977 del INE

\section{TABLA 10}

Proporción de mujeres casadas que controlan la natalidad, según sus estudios y deseos de embarazo

\begin{tabular}{|c|c|c|}
\hline \multirow[b]{2}{*}{ Estudios de la mujer } & \multicolumn{2}{|c|}{$\begin{array}{l}\% \text { de mujeres casadas que } \\
\text { controlan la natalidad }\end{array}$} \\
\hline & $\begin{array}{c}\text { Si desean otro } \\
\text { embarazo }\end{array}$ & $\begin{array}{c}\text { No desean otro } \\
\text { embarazo }\end{array}$ \\
\hline Superiores ............. & $63 \%$ & $55 \%$ \\
\hline 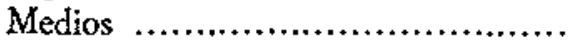 & $54 \%$ & $73 \%$ \\
\hline 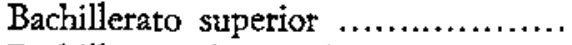 & $66 \%$ & $63 \%$ \\
\hline Bachillerato elemental ................ & $49 \%$ & $65 \%$ \\
\hline Primaria $\ldots \ldots \ldots \ldots \ldots \ldots \ldots \ldots \ldots \ldots \ldots \ldots \ldots \ldots \ldots$ & $53 \%$ & $53 \%$ \\
\hline Sin estudios ${ }^{*} \ldots$ & $43 \%$ & $49 \%$ \\
\hline \multirow[t]{2}{*}{ Total ... } & $52 \%$ & $54 \%$ \\
\hline & $(758)$ & $(1.801)$ \\
\hline
\end{tabular}

Nota: a) Se inciuyen en este caso también las canalfabetass.

Fuente: INE, Encuesta de fecundidad (Madrid: INE. 1978), pp. 217-219.

\section{TABLA 11}

Métodos de contracepción utilizados según los estudios de la mujer

$\%$ de mujeres casadas (y no embarazadas) que actualmente utilizan algún método de contracepción

\begin{tabular}{|c|c|c|c|c|c|c|}
\hline Estudios de la mujer & $\begin{array}{c}\text { Coito } \\
\text { interrumpido }\end{array}$ & Pildora & Ritmo & Condon & $\begin{array}{l}\text { Otros } \\
\text { métodos }\end{array}$ & Total \\
\hline Superiores & $0,0 \%$ & $41,0 \%$ & $8,8 \%$ & $2,9 \%$ & $7,4 \%$ & $60 \%$ \\
\hline Medi & 5,5 & 27,0 & $14,0 \%$ & & & $57 \%$ \\
\hline lerato superior. & $5,2 \%$ & $22,0 \%$ & $15,0 \%$ & $6,5 \%$ & $7,8 \%$ & $57 \%$ \\
\hline Bachillerato elemental & $8,9 \%$ & $17,0 \%$ & $12,0 \%$ & $4,8 \%$ & $8,6 \%$ & $51 \%$ (392) \\
\hline Primaria $\ldots \ldots \ldots \ldots \ldots$ & $22,0 \%$ & $10,0 \%$ & $5,8 \%$ & & $3,0 \%$ & $46 \%(3.353)$ \\
\hline Sin estudios ............ & $27,0 \%$ & $6,2 \%$ & $1,8 \%$ & $1,9 \%$ & $3,7 \%$ & $41 \%(1.053)$ \\
\hline Analfabetas $\ldots \ldots \ldots \ldots$ & $26,0 \%$ & $3,9 \%$ & $0,4 \%$ & $3,7 \%$ & $2,7 \%$ & $3790 \quad(515)$ \\
\hline Total & $21,0 \%$ & $11,0 \%$ & $5,7 \%$ & $4,5 \%$ & $3,8 \%$ & $46 \%(5.810)$ \\
\hline
\end{tabular}

Nota: Son datos referentes a mujeres casadas actualmente y expuestas al riesgo del embarazo. Véanse también las notas de las Tablas 4 y 6 .

Fuente: INE, Encuesta de fecundidad (Madrid; INE. 1978), p. 170. 
«Qapers»: Revista dt Suciologis

\section{TABLA 12}

Métodos de contracepción utilizados según la clase social

\begin{tabular}{|c|c|c|c|c|c|c|}
\hline \multirow[b]{2}{*}{$\begin{array}{c}\text { Clase social } \\
\text { (según la ocupación } \\
\text { del marido) }\end{array}$} & \multicolumn{6}{|c|}{$\begin{array}{c}\% \text { de mujeres casadas (y no embarazadas) que actualmente } \\
\text { utilizan algún método de contracepción }\end{array}$} \\
\hline & $\begin{array}{l}\text { Coito } \\
\text { interrum- } \\
\text { pido }\end{array}$ & Pildora & Ritmo & Condón & $\begin{array}{l}\text { Otros } \\
\text { métodos }\end{array}$ & Total \\
\hline \multicolumn{7}{|l|}{ URBANA: } \\
\hline Empresarios $" . . . . . . .$. & $18,0 \%$ & $5,1 \%$ & $7,6 \%$ & $3,2 \%$ & $3,8 \%$ & $37 \% \quad(158)$ \\
\hline Cuadros superiotes. & $4,8 \%$ & $24,0 \%$ & $16,0 \%$ & $4,1 \%$ & $4,8 \%$ & $54 \% \quad(315)$ \\
\hline Cuadros medios .... & $14,0 \%$ & $18,0 \%$ & $11,0 \%$ & $6,3 \%$ & $6,3 \%$ & $55 \%(1.129)$ \\
\hline Trabajadores indepen- & & & & & & \\
\hline dientes ............... & $27,0 \%$ & $8,6 \%$ & $4,8 \%$ & $4,6 \%$ & $3,9 \%$ & $49 \% \quad(544)$ \\
\hline Obreros especializados & $25,0 \%$ & $9,5 \%$ & $3,5 \%$ & $5,2 \%$ & $3,0 \%$ & $46 \%(2.192)$ \\
\hline $\begin{array}{l}\text { Peones (obreros no } \\
\text { especializados) }\end{array}$ & $23,0 \%$ & $6,1 \%$ & $1,2 \%$ & $4,9 \%$ & $2,2 \%$ & $37 \%$ \\
\hline \multicolumn{7}{|c|}{ RURAL: } \\
\hline Propietarios ${ }^{b} \ldots \ldots \ldots$ & $19,0 \%$ & $3,0 \%$ & $5,1 \%$ & $0,3 \%$ & $1,2 \%$ & $28 \%$ \\
\hline Jornaleros ........... & $28,0 \%$ & $1,7 \%$ & $1,4 \%$ & $1,2 \%$ & $4,3 \%$ & $37 \% \quad(419)$ \\
\hline Total $\mathrm{F}, \ldots \ldots \ldots$ & $21,0 \%$ & $11,0 \%$ & $5,7 \%$ & $4,5 \%$ & $3,8 \%$ & $46 \%(5.814)$ \\
\hline
\end{tabular}

Notas: a) En las tablas originales se trata de aempresarios con asalariados», pero no se distingute si son urbanos o rurales.

b) :Empresarios agrarios sin asalariados y miembros de cooperativas agrarias».

c) Incluye 231 casos de wactivos no clasificabless.

Fuente: INE, Encuesta de fecundidad (Madrid: INE, 1978), p. 176.

\section{TABLA 13}

Métodos de contracepción utilizados según los estratos de población

\begin{tabular}{|c|c|c|c|c|c|}
\hline \multirow[b]{2}{*}{$\begin{array}{c}\text { Métodos que } \\
\text { utilizan actualmente } \\
\text { de contracepción }\end{array}$} & \multicolumn{5}{|c|}{ Tamaño del municipio } \\
\hline & $\begin{array}{l}\text { Grandes } \\
\text { ciudades }\end{array}$ & $\begin{array}{l}\text { Más de } \\
50.000 \\
\text { babitantes }\end{array}$ & $\begin{array}{c}\text { De } 10.000 \\
\text { a } 50.000 \\
\text { babitantes }\end{array}$ & $\begin{array}{c}\text { Hasta } \\
10.000 \\
\text { babitantes }\end{array}$ & Total \\
\hline Coito interrumpido. & $15,0 \%$ & $21,0 \%$ & $23,0 \%$ & $23,0 \%$ & $21,0 \%$ \\
\hline Píldora ................ & $23,0 \%$ & $11,0 \%$ & $9,1 \%$ & $3,4 \%$ & $11,0 \%$ \\
\hline Ritmo ................. & $1,4 \%$ & $75 \%$ & $3,5 \%$ & $4,0 \%$ & $5,7 \%$ \\
\hline Condón...$\ldots \ldots \ldots \ldots$ & $4,2 \%$ & $6,2 \%$ & $3,7 \%$ & $3,0 \%$ & $4,5 \%$ \\
\hline Otros métodos ....... & $5,6 \%$ & $4,5 \%$ & $3,4 \%$ & $2,2 \%$ & $3,8 \%$ \\
\hline Total ............... & $\begin{array}{c}56,0 \% \\
(943)\end{array}$ & $\begin{array}{c}50,0 \% \\
(2.106)\end{array}$ & $\begin{array}{c}42,0 \% \\
(1.259)\end{array}$ & $\begin{array}{c}36,0 \% \\
(1.507)\end{array}$ & $\begin{array}{l}46,0 \% \\
(5.814)\end{array}$ \\
\hline
\end{tabular}

Fuente: INE, Encuesta de fecundidad (Madrid: INE, 1978), p. 169. 
La encuesta de fecundidad de 1977 del INE

\section{TABLA 14}

Métodos de contracepción utilizados en las diversas tegiones

\begin{tabular}{cccccccc}
\hline \multicolumn{7}{c}{$\%$ de mujeres casadas (y no embarazadas) que actualmente } \\
utilizan algún método de controcepción
\end{tabular}

Fuente: INE, Encuesta de fecundidad (Madrid: INE, 1978), p. 168. 